\title{
Flexible Linear Polyelectrolytes in Multivalent Salt Solutions: Solubility Conditions
}

\author{
F. J. Solis and M. Olvera de la Cruz \\ Northwestern University, Department of Materials Science and Engineering \\ Evanston, IL 60208, USA \\ e-mail:f-solis@northwestern.edu, m-olvera@northwestern.edu
}

\begin{abstract}
Polyelectrolytes such as single and double stranded DNA and many synthetic polymers undergo two structural transitions upon increasing the concentration of multivalent salt or molecules. First, the expanded-stretched chains in low monovalent salt solutions collapse into nearly neutral compact structures when the density of multivalent salt approaches that of the monomers. With further addition of multivalent salt the chains redissolve acquiring expanded-coiled conformations. We study the redissolution transition using a two state model [F. Solis and M. Olvera de la Cruz, J. Chem. Phys. 112 (2000) 2030]. The redissolution occurs when there is a high degree of screening of the electrostatic interactions between monomers, thus reducing the energy of the expanded state. The transition is determined by the chemical potential of the multivalent ions in the solution $\mu$ and the inverse screening length $\kappa$. The transition point also depends on the charge distribution along the chain but is almost independent of the molecular weight and degree of flexibility of the polyelectrolytes. We generate a diagram of $\mu$ versus $\kappa^{2}$ where we find two regions of expanded conformations, one with charged chains and other with overcharged (inverted charge) chains, separated by a collapsed nearly neutral conformation region. The collapse and redissolution transitions occur when the trajectory of the properties of the salt crosses the boundaries between these regions. We find that in most cases the redissolution occurs within the same expanded branch from which the chain precipitates.
\end{abstract}

\section{INTRODUCTION}

The precipitation and dissolution of linear polyelectrolytes with the addition of multivalent salt or particles have been extensively studiedll 6 . In DNA the precipitation provides a promising mechanism to "pack" long DNA, a critical problem in gene therapy. Moreover, it is correlated with highly accelerated rates of DNA renaturation and cyclisation 19,11 . Therefore, it is important to determine the concentration of multivalent salt or particles at which the precipitated DNA dissolves in the solution. Furthermore, it is crucial to determine the effective charge of the DNA in the precipitated and dissolved states to determine their interaction with cells.

It has been shown experimentally that the transitions are nearly independent of the details of the linear polyelectrolyte (such as charge density, degree of flexibility, molecular weight, structure function, etc.) 2. That is, flexible (single stranded DNA and Polystyrene Sulphonate) and semiflexible chains (double stranded DNA) can be described by the same thermodynamic model even though flexible chains collapse into amorphous dense spheres and long semiflexible chains into toroid conformations 12 .

We recently develop a thermodynamic model that explains the universal nature of the multivalent counterion induced precipitation transition in low concentration of monovalent saltst. In this paper we analyze the redissolution transition by extending our two state model to include large concentrations of multivalent salt.

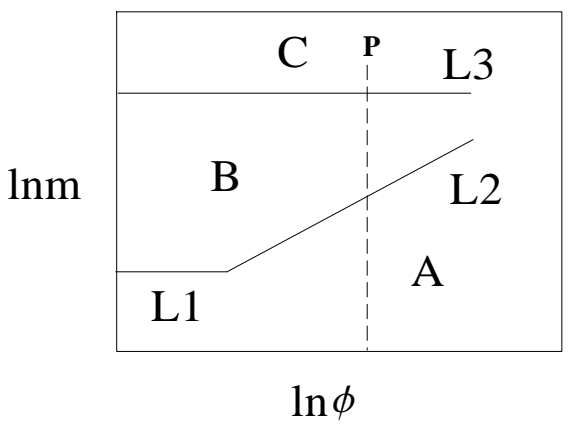

FIG. 1. Location of the collapse and redissolution transitions in a diagram of the logarithm of the concentrations of monomers $\phi$ and of multivalent salt $m$, for dilute, strongly charged polyelectrolytes. Lines L1 and L2 form the boundary between the expanded-stretched state and the collapsed state, and line L3 is the boundary between collapsed and expanded-coiled states. The line $\mathrm{P}$ is a guide used in the text and represents the increase of multivalent salt at fixed monomer concentration.

The experimental diagrams of the logarithm of concentration of monomers $\phi$ versus the logarithm of the concentration of multivalent salt $m$ have three regions (A, B anf C) separated by 2 transitions lines (L1-L2 and L3)2 3 , shown schematically in Fig. 1. Region A corresponds to polyelectrolytes dissolved in water with expanded-stretched conformation at low $m$ values. Region $\mathrm{B}$ at intermediate multivalent salt concentrations $m$ is a solution of collapsed polyelectrolytes (sometimes considered as a coexistence of two phases: one rich and one poor in polyelectrolytes). Region $\mathrm{C}$ at large $m$ values contains polymers dissolved in water in expanded- 
coiled conformations. The transition line between the $\mathrm{A}$ and $\mathrm{B}$ regions, L2, at low monovalent salt concentrations is linear with a slope comparable to $1 / z$, where $z$ is the valence of the counterions of the added salt. In the presence of monovalent salt there is a nearly horizontal transition line, L1, at very low concentrations of monomers. The transition line between regions $\mathrm{B}$ and $\mathrm{C}$ is horizontal (i.e., independent of the concentration of monomers) in the full regime. In our previous paper we discussed the transition between regions $\mathrm{A}$ and $\mathrm{B}$ and explained the transition in the regime L2 as the creation of collapsed conformations of polymers whose charge is compensated by condensed multivalent salt. Our model also predicts the transition L1 in the presence of large amounts of monovalent salt. In this paper we study the transition between the regions $\mathrm{B}$ and $\mathrm{C}$ in solutions with very low (negligible) monovalent salt concentrations.

It is well documented that in polyelectrolyte solutions a fraction of the counterions condensed along the polyelectrolytes to decrease their electrostatic energy 14 . This ion condensation is crucial to understand the precipitation and redissolution transitions. In this paper we compute the fraction of condensed ions as the multivalent salt concentration increases in the two possible polyelectrolyte conformations: collapsed and expanded. Our two state model is based on the fact that in salt free and/or low ionic strength solutions, ion condensation leads to two possible conformation in linear flexible polyelelctrolytes 13: expanded-stretched with a reduced effective charge and collapsed nearly neutral with the cohesive energy of an ionic glass. Indeed, multivalent counterions lead to large cohesive energies for nearly neutral collapsed structures in region B explaining the transition L2. Since the magnitude of the electrostatic energy of a collapsed chain in region B is much larger than any entropic energy increase (due to the decrease of degrees of freedom resulting from the compaction), our model also describes the precipitation of semiflexible and rigid-rod polyelectrolytes into toroids and bundles, respectively, explaining the universality of the precipitation transition discussed in our previous workt.

At the precipitation transition we predict that the expanded chains are slightly charged, and with further addition of multivalent salt, the collapsed chains (in region B) become practically neutral, in excellent agreement with recent electrophoresis experiments 3 . As a result, the aggregates interactions in the dilute regime are negligible justifying a monomolecular collapse model to describe also multi-molecular aggregation.

The effective charge and size of the expanded conformation (in region $\mathrm{C}$ ) changes with further addition of multivalent salt. The transition L3 occurs at high salt concentrations, and in such conditions the chains are expected to obey random walk or self-avoiding walks statistics due to the screening of the electrostatic interactions 15 . Screening is responsible of the redissolution, as suggested in various models 10 . We show here that the redissolution is actually very sensitive to the re- lation between the chemical potential of the condensing multivalent particles in the solution $\mu$ and the inverse screening length $\kappa$, while nearly insensitive to the degree of flexibility and molecular weight of the polyelectrolytes. Screening, for example, may be reduced due to multivalent-monovalent ion associating in the solution, strongly affecting the effective charge of the expanded chains. We determine the effective charge of the chains as a function of $\mu$ and $\kappa$ including the finite size of the ions and the discrete nature of the charge distribution along the polyelectrolyte, which is essential to compute the electrostatic energies of both the collapsed and expanded stated In a $\mu-\kappa$ diagram the collapsed region lies between two branches of expanded states: one expanded with a reduced effective charge and the other expanded with an inverted charge. In most common situations the chains precipitate and redissolve within the same branch; i.e., with a reduced effective charge of the same sign of the bare charge. Our model predicts a redissolution transition independent of polyelectrolyte concentration and describes the re-dissolution of flexible, semiflexible and rigid polyelectrolytes. Indeed, the collapsed and expanded states discussed here are akin to the multi-molecular precipitated and dissolved states, respectively, observed in many polyelectrolytes3, explaining the rather universal form of the diagram, Fig. 1.

In the Section 2 we summarize the assumptions of our work, and provide a description of each of the phases of the system. In section 3 we construct the free energies of two types of representative states and in Section 4 we give the result of the free energy minimization. In Section 5 we summarize our results, compare them with experiments and emphasize the elements of the theory that have not yet been subject to experimental results. We end with a brief conclusion in section 6 .

\section{THEORETICAL MODEL}

As in our previous workd, we rely in a two state description of the system. Instead of considering all possible states of the system (describing them, for example by a changing scaling exponent), we simply assume that the minimum lies in one of two extremes: collapsed or expanded conformations. The expanded conformation, however, is a function of the concentration of salt in the solution. For example, at low monovalent salt concentrations the chains are expanded rofs, and at large concentrations they are expanded coils 5 . The free counterions and solvent can be integrated out provided we take into account the electrostatic interactions between the noncondensed counterions in the solution.

The condensed (collapsed) state is treated as an ionic glass so that its energy can be approximately calculated by techniques from solid state physics17; i.e, we assume that fluctuations play no important role and can be neglected. For a very large chain, at low enough tempera- 
tures, the bulk of the collapsed state should acquire an almost crystalline structure. The finite size of the chain, the connectivity of the monomers, and finite temperature effects must induce defects into the structure and the local structure should be glass-like, where the perfect order has not been achieved. An ionic glass structure was indeed observed in recent numerical studies of polyelectrolytes in bad solvents 16 .

In both states of the systems, the collapsed and expanded states, it is necessary to keep track of the finite size of the counterions and monomers. This is clear in the case of the collapsed state, but even in the expanded states there is an important contribution arising from interactions with the nearest neighbors, and this energy can only be calculated by considering the finite size of the particlest. For example, a naive coarse-grained model that takes into account only effective charges would assign a zero energy to a neutral cluster, while it can actually have a very strong cohesive energy. The redissolution into an expanded state occurs at high salt concentrations and the effective interactions between monomers are screened. In these conditions the energy associated with interactions between far away segments of the chain can be treated as a small perturbation with respect to that of the neutral polymer state.

The properties of the salt at different concentrations are summarized into two parameters: an effective chemical potential $\mu$, and an inverse screening length $\kappa$. We will present our results for general values of these parameters and in particular consider the case were the parameters are related by a Debye-Huckel law. We also discuss the effect of multivalent-monovalent ion association, the Bjerrum model18, in the diagram.

Let us consider the series of states of a single polymer chain along a line of constant polymer concentration (line $\mathrm{P}$ in Fig. 1) with increasing multivalent salt concentration. We start in a state in region $\mathrm{A}$ with few counterions condensed. The condensed counterions lie very close to the chain, and cannot be considered as mixed in the solvent. The effective charge induces repulsions between the segments of the chain, and the chain acquires extra stiffness, making it into a rod-like structure.

When a small quantity of multivalent salt is added to the system, it dissociates, and the multivalent counterions are absorbed to the chain because the electrostatic energy to be gained from the condensation is larger (per unit charge) than that of the monovalent counterions. Furthermore, the entropic loss due to condensation is lower for the multivalent counterions. When the charge of the added salt roughly equals the bare charge of the polymers the effective charge of the chains quickly goes to zero. At this point most charges of the chain are compensated by the condensed multivalent counterions and a few monovalent ones. Since a group of charges with total charge zero has a minimal energy when they are set in a compact configuration, a collapsed conformation is acquired. This transition is of course also possible for the case of pure monovalent salt, but it requires either larger concentrations or lower temperatures to offset the entropic contributions. In other words, the determining factor for the transition is the strength of the electrostatic interaction, and the introduction of multivalent salt acts as a jump in parameter space and is not equivalent to a smooth increase in monovalent salt

In the collapsed state the absence of uncompensated charges in the polymer reduces its solubility in water and polar solvents. It is not clear from experiments, however, whether the collapsed flexible polymers aggregate into large structures or if they form a dense solution of individually collapsed chains. Though we assume monomolecular collapse, the predicted transition points should not exhibit strong dependence on the number of chains aggregated since if the collapsed state is neutral, the residual interactions between chains (promoting or opposing multimolecular aggregates) are energetically small.

Since the collapsed structure is almost neutral, the increase in salt concentrations has little effect in the absolute value of the energy of the chain. The change in the environment affects only the interaction between the positive or negative small excess (or defect) of charge in the polymer, which would be located at the surface of the collapsed structure. The energy of the charges packed inside the structure is dominated by the interaction with their nearest neighbors and is not subject to screening (there are no floating ions between them). On the other hand, the energy of an expanded chain with non-zero effective charge will be greatly reduced by the increased screening.

For the purposes of comparison with the collapsed structures, we can consider a very rigid chain that cannot be collapsed into a compact structure. We can ignore for the moment the fact that this chain would likely form a bundle with other chains, and we can assume that we have isolated it by perhaps mechanical means. At higher multivalent salt concentrations, the rigid polymer continues absorbing ions, and thus it can become neutral and even overcharged (effective charge with opposite sion fo the bare charge, as it occur in many systems20,19.21 23). With further increase in the amount of salt the trend can be reversed, and the chain can again become, first neutral, and then simply charged (same charge sign as the bare charge). The increase of salt concentration reduces the entropic penalty for the condensation of counterions. The counterions continue condensing into the polymer as long as there is a reduction in electrostatic energy due to the condensation. The increase in condensation stops when the typical screening length of the free counterions is of the order of the monomer separation, the free counterions can reduce their energy by associating with each other, and a fraction of them will do so instead of associating with the polymer.

At large salt concentrations, the chain reverts from collapsed to expanded, giving the transition $B-C$. In the expanded state the chain needs not to be neutral. The screening of electrostatic interaction under these condi- 
tions strongly reduces the interaction between the uncompensated charge of the polymer, and it is clear that the repulsions will not be strong enough to create a rodlike state. In these conditions an expanded coil state is more likely. In terms of the effective charge of the polymer we find that two scenarios are in principle possible. If the chemical potential of the counterions in the solvent is low enough while the screening length is reduced, the expanded state is overcharged since there is an effective decrease of energy by increasing the condensation to the chain, as recently discussed by Nguyen et al.8. On the other hand, when the screening length is small, it is possible that the interactions between coions and counterions in the solvent become important, and it is much harder for the chain to acquire the counterions. This is, the chemical potential for the extraction of counterions from the solvent is large and therefore the effective charge at the redissolution point has the same sign as the bare charge. We show in the next section that though there is a possibility for overcharged polyelectrolytes at the redissolution, in the sense that this state can be preferred to a collapsed one, the salt properties required for this situation $(\mu, \kappa)$ are unlikely to be realized in practice for water soluble multivalent ions.

\section{CALCULATION OF FREE ENERGIES}

We assumed that the polymers are monodisperse with monomer number $N$. Each monomer is charged positively, and is monovalent. The original counterions of the polymer also have valence 1 . The polymer density is $\phi$. We measure all distances with respect to the monomer size $b$ (so that $b=1$ in our units). It is necessary for more precise calculations to also give more detailed geometrical information such as the aspect ratio of the monomers and the respective size of all the ions. Here we only need to further consider, besides the size of the monomer, the radius of the multivalent counterions $a$. The monovalent salt concentration $s$ is set equal to zero, but there are monovalent counterions originally dissociated from the polymer with density $\phi$, and the multivalent salt, with valences $z: 1$ has concentration $m$. All energies are measured in units of $k_{B} T$, (the Boltzmann constant times the temperature), and consider only energies per monomer. We form the dimensionless Bjerrum number $B$, equivalent to the Manning parameter, as

$$
B=\frac{e^{2}}{4 \varepsilon_{0} \pi b k_{B} T}
$$

where $\varepsilon_{0}$ is the permitivity of the solvent (water). The typical value of this number at room temperature in strongly charged polyelectrolytes is larger than 1 and as high as 4.2 for double stranded DNA. We assume here for simplicity that $B$ is a constant, independent of the number of locally associated monomers and ions. Given that in both states water is always present, the corrections in the values of $\epsilon$ are expected to be minor 24 .

In both expanded and collapsed states we describe the system by a particular geometry and a given fraction $x$ of condensed charge. The free energy is separated into a contribution from the solvent and free ions, and another part from the interaction of the monomers and charges condensed to them. The fraction condensed is obtained self-consistently from minimization over all possible condensed fractions. The form of the entropic term is the same in both cases, but it is easier to consider the electrostatic contributions separately.

\section{A. Free counterions contributions}

It is convenient to separate the region occupied by the polymer from the rest of the solvent. To calculate the free energy of the solvent region, we first calculate the entropic contribution. We assume that the charge condensed to the chain $-x N$ is composed by $x_{s} N$ monovalent ions and $x_{m} N / z$ multivalent ions. Thus there are $(s / \phi)+1-x_{s}$ free monovalent counterions per monomer, and thus the energy per monomer in $k_{B} T$ units is

$$
F_{s 1}=\left(\frac{m}{\phi}-\frac{x_{m}}{z}\right) \ln \left(m-x_{m} \frac{\phi}{z}\right)+\left(\frac{s}{\phi}+1-x_{s}\right) \ln \left(s+\left(1-x_{s}\right) \phi\right) \text {. }
$$

In this expression we omitted the contribution of the ions with the same charge of the monomers, since it is assumed that they do not condense, and thus have a constant contribution. We have also omitted the contribution of the solvent, since the concentration of the salts is small compared to the solvent. It is possible to add in this expression an interaction term between the solvent and the ions, but for simplicity we neglect these corrections here; its effect would be only to shift the position of the L3 line in the phase diagram. It is important to consider, however, the effective free energy of interaction between all free ions. A simple way to do this is to use the modified Debye-Huckel form:

$$
F_{s 2}=\frac{1}{4 \pi \phi}\left(\ln (1+\kappa a)-\kappa a+\frac{1}{2}(\kappa a)^{2}\right) .
$$

In this expression $a$ is the hard-core radius of the salt ions and $\kappa$ is the inverse screening length produced by the salt:

$$
\kappa^{2}=4 \pi B\left(z^{2}\left(m-x_{m} \phi / z\right)+z m+\left(2 s+\left(1-x_{s}\right) \phi\right)\right) .
$$

In the very dilute polymer limit, we can simplify these expressions, and use instead

$$
\kappa^{2}=4 \pi z(z+1) B m
$$

and expand the total free energy of the solvent $F_{s}=$ $F_{s 1}+F_{s 2}$ in a series in the overcharge fraction $y=x-1$. 
The constant term is irrelevant and we retain only the linear term:

$$
F_{s}=\left(\partial_{y} F_{s}\right) y=\mu y,
$$

where $\mu$ is now the effective chemical potential for the extraction of ions from the solvent into the condensed region. Using the same high salt limit as before we obtain:

$$
\mu=-\frac{1}{z} \ln m+\frac{1}{8 \pi(z+1) m} \frac{\kappa^{3}}{1+\kappa a} .
$$

\section{B. Expanded state energy}

In our previous work of rod-like conformations. Since our interest is the regime with large amounts of salt, the screening strongly reduces the rigidity of the chain due to excess charge. It is then adequate to consider a Gaussian conformation (selfavoiding walk conformations give very similar results, as it can be seen from the functional form of the free energy computed below). The electrostatic contribution to the free energy is calculated as a function of the condensed charge, and we set the neutral state $x=1$ as reference. The free energy is expressed as the sum of three terms. A constant term reflects the energy of the neutral state, a term linear in the excess charge $y=(x-1)$ appears due to addition or extraction of a charge into or from the neutral state. Finally, the interaction between segments of the chain with excess charge gives rise to a term quadratic in $y$. We write this as:

$$
F_{e}^{e}=g_{0}^{e}+g_{1}^{e} y+g_{2}^{e} y^{2}
$$

A coarse-grained model clearly gives rise to the last term, and the first two can be understood, in that context, as regularizations that take into account the finite size of the particles involved. In the limit of large amounts of saltadded, most of the condensed charge comes from multivalent ions, and thus, we will take $x=x_{m}$, and $x_{s}=0$. The terms are graphically represented in Fig.2.

For the calculation of the energy of the neutral state we basically apply the Wigner principle 17: in a dense ionic system, the free energy can be approximated by the contribution of nearest neighbors that effectively cancel the charge of the particle. In the case of the collapsed state, below, it is more suitable to consider an approximation based on the calculation related to a full infinite lattice.
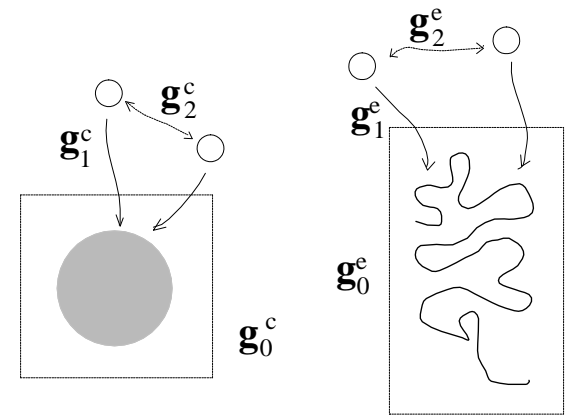

FIG. 2. Graphical representation of the terms required to calculate the energy of the system in both states. The $g_{0}$ term measures the energy per monomer of the chain in a neutral state. $g_{1}$ measures the energy of adding an additional charge to the neutral conformation. $g_{2}$ is the energy of interaction between added charges and is obtained by a coarse graining procedure. All the excess charge in the collapsed state (b) is assumed to lie on its surface.

The basic neutral cluster of the linear polyelectrolyte consists of one multivalent counterion, and $z$ monomers. Since all particles in the cluster (with charges $q_{I}$, both monomers at distances $r_{i j}$ from each other, all roughly equal to the monomer size $r=b=1$, the electrostatic energy of the cluster is

$$
E=\sum_{i \neq j} B \frac{q_{i} q_{j}}{r_{i j}}=-B\left(z^{2}+z(z-1) / 2\right)
$$

Dividing by $z$ monomers, we obtain:

$$
g_{0}^{e}=-B(z+1) / 2 .
$$

When an extra multivalent ion is added to this cluster, the counterion can have roughly the same interaction with the chain monomers, but can locate itself away of the first counterion, say, by putting a monomer in between them so that $r \approx 2$. Thus we obtain a simple estimate for the energy of the new charged cluster as:

$$
E=B\left(-2 z^{2}+z(z-1) / 2+z^{2} / 2\right),
$$

and

$$
g_{1}^{e}=-B z / 2 .
$$

Finally, the two-body term can be calculated assuming a uniform distribution of charge along the Gaussian chain. A Gaussian chain has a monomer distribution given, approximately, by $\rho(r)=1 /(2 \pi r)$, inside the volume $V$ limited by the radius of gyration $R$; this is, for $r<R=N^{1 / 2}$. A charge at the center of this distributions interacts with other charges via the screened potential $B e^{-\kappa r} / r$. The energy for one such charge is given by the average over the volume $V$ of this potential:

$$
E=\langle V\rangle=\int_{V} d \mathbf{r} \rho(r) B \frac{e^{-\kappa r}}{r}=\frac{B}{\kappa}\left(1-e^{-\kappa R}\right),
$$


and by our previous definition,

$$
g_{2}^{e}=\frac{1}{2}\langle V\rangle
$$

Notice that while this calculation depends on the assumed Gaussian conformation, a simple estimate can also be done by using a general expansion coefficient for the radius of gyration $R \approx N^{\nu},(\nu=1$ for rod-like, $\nu=2 / 3$ for self-avoiding walks, and $\nu=1 / 2$ for random walks). In such case, we obtain a scaling for the quadratic precoefficient of the form $g_{2}^{e} \approx \kappa^{(1-1 / \nu)}$. While these different models give rise to different estimates, the transition we are interested in occurs when the screening is of the order of the size of the monomers, and so, in our units, $\kappa \approx 1$, and the estimates at that point are not so different.

\section{Collapsed state energy}

We repeat a similar approach for the calculation of the free energy of the collapsed state which is expressed as:

$$
F_{e}^{c}=g_{0}^{c}+g_{1}^{c} y+g_{2}^{c} y^{2} .
$$

Inside the collapsed polymer, we can imagine the environment of a charge resembling that of a ionic crystal. If the structure of the crystal is known, the energy per atom in that crystal can be given in terms of the Madelung constant 25

$$
E=-M e^{2} / r=-M B .
$$

The Madelung constant for an ionic monovalent salt is $M=-1.747$. There are two important differences in our case. We would like to consider multivalent ions, which reduce the contribution from same-charge interactions, and on the other hand the connectivity of the monomers forces them to be next-neighbors (not the case in ionic crystals), and thus increases the same-charge interactions. A way to obtain a rough estimate for $M$ is to consider a simple geometry in which both monomers and counterions lie in columns which in turn form a square lattice when a cross section is taken. The result does not change dramatically when other possible geometries are considered. As a rule of thumb, we can use an effective Madelung constant that is larger than unity, but not much bigger. In the geometry proposed, the number of next neighbors of counterion is $4 z$. Summing the interaction with $4 z$ monomers and 4 equally charged nextnearest counterions $(r=2 b)$ we obtain the following estimate of the energy per cluster of 1 counterion and $z$ monomers:

$$
E=B\left(-4 z^{2}+z(z-1)+\frac{1}{2} z(4 z-z)+\frac{1}{2} \frac{4 z^{2}}{2}\right)
$$

where the interactions with charges outside the cluster are weighted by $1 / 2$ to avoid double counting. Dividing again by $z$, we obtain the coefficient of the energy per monomer:

$$
g_{0}^{c}=-B\left(1+\frac{z}{2}\right) .
$$

In this approximation we have underestimated the Madelung constant for $z=1$, for which we obtain -1.5 instead of the exact result -1.747 , but it is a reasonable approximation and shows that the energy of this neutral collapsed state is smaller than the neutral expanded state.

In both the collapsed and expanded states, the addition and extraction of charges are not completely symmetric processes. It is energetically unfavorable to add or extract charges from the collapse bulk state, as shown below in the calculation of $g_{2}^{c}$. Both processes are however better thought of as occurring at the surface of the collapsed region. We have also argued previouslyd, that while the mobility of charges in this type of conformations is not so large, the analogy with a conductor is still useful: all charge excess resides in the surface. While the local conformation at the surface of the collapsed state is not equal to the expanded state, it is simpler to borrow the result for the coefficient related to the addition of one charge from that case. In both situations the new charge is put in close contact with a neutral cluster, but sits slightly closer to the opposite charges. Thus,

$$
g_{1}^{c}=-B z / 2 .
$$

Since we assume that the extra charge is sitting at the surface, the interaction energy can be calculated assuming a uniform distribution in a shell surrounding the collapsed polymer. We assume this shell to be spherical with radius $R$, and obtain the energy per added charge as

$$
g_{2}^{c}=E=\frac{1}{2} B \frac{1}{R}=\frac{1}{2} \frac{B}{(3 N(1+1 / z) / 4 \pi)^{1 / 3}}
$$

Here we have considered that the $N+N / z$ charged particles are closely packed and that they occupy each a volume of $b^{3}$.

\section{COMPARISON BETWEEN STATES AND PHASE DIAGRAM}

After the minimization of the free energies of each state with respect to their respective condensed charge variables, we can compare each other to find the state of the chain for given concentration conditions. This minimization can be done directly from the expressions already given but it is easier to present simpler formulas if we use a set of approximations described below. Numerical results presented later reflect these approximations, but we have checked that numerical minimization of the full expressions do not lead to important qualitative and quantitative changes in the results. 
The free energy of each of the states, for given concentrations of monomers and salt correspond to the minima of:

$$
\begin{aligned}
& F^{e}=F_{s}+F_{e}^{e}, \\
& F^{c}=F_{s}+F_{e}^{c}
\end{aligned}
$$

for the expanded and collapsed states respectively. We minimize now with respect to the overcharge fraction $y$.Taking the first derivatives with respect to this quantity, we obtain the (independent) conditions

$$
\begin{aligned}
& \partial_{y} F^{e}=\mu+g_{1}^{e}+2 g_{2}^{e} y^{e}=0, \\
& \partial_{y} F^{c}=\mu+g_{1}^{c}+2 g_{2}^{c} y^{c}=0 .
\end{aligned}
$$

The solutions for the fractions condensed are:

$$
\begin{aligned}
& y^{e}=-\left(\mu+g_{1}^{e}\right) / 2 g_{2}^{e}, \\
& y^{c}=-\left(\mu+g_{1}^{c}\right) / 2 g_{2}^{c} .
\end{aligned}
$$

Replacement of this values in the general expressions for the free energy gives the minimized free energies for each state:

$$
\begin{aligned}
& F^{e *}=F^{e}\left(y^{e}\right)=\mu+g_{0}^{e}-\frac{\left(g_{1}^{e}+\mu\right)^{2}}{4 g_{2}^{e}}, \\
& F^{c *}=F^{c}\left(y^{c}\right)=\mu+g_{0}^{c}-\frac{\left(g_{1}^{c}+\mu\right)^{2}}{4 g_{2}^{c}} .
\end{aligned}
$$

For the moment it is convenient to maintain the expressions of the free energies as functions of the chemical potential instead of the salt concentration. Of the parameters used to describe the different states, only $g_{2}^{e}$ has an explicit dependence on the concentration (through the inverse screening length $\kappa)$ l. Also, it is important to note that the coefficient $g_{2}^{c}$ is extremely large, and for the values of the chemical potential that we need to explore, it turns out that the denominator in the expression for the overcharge is much larger than the numerator, and the overcharging fraction is very close to 0 :

$$
y^{c} \approx 0
$$

This result is clear since we have found that there is a very strong penalty for any overcharging (or undercharging) in the collapsed state. The effective charge in a general structure is affected by the environment (namely, by $\mu$ ) but the collapsed structure is only very slightly susceptible to it. Furthermore, the interaction between the charges in the bulk of the collapsed structure is not subject to screening by the free particles outside and its contribution remains constant with changing chemical potential. Thus, the system splits neatly into collapsed neutral structures and a homogeneous mixture of solvent and non-condensed counterions. It is then not surprising that this state adequately represents what in experiment is close to be two separate phases.

Consider now the minimum for the expanded state. The coefficient for charge-charge interactions $g_{2}^{e}$ varies very strongly with the salt concentration. It remains large when the screening length is large, since in those conditions all uncompensated charges interact strongly. On the other hand at large concentrations of salt the screening drastically reduces the interaction between segments of the chain.

To obtain an expression for the transition point we subtract the free energies, and define the energy difference

$$
\Delta F=F^{e *}-F^{c *} .
$$

The system is in the region $B$, when $\Delta F>0$, in region $C$ when $\Delta F<0$, and the transition curve is defined by $\Delta F=0$. Using the fact, discussed above, that the electrostatic energy of the collapsed state remains essentially constant, we can write the equation $\Delta F=0$ as

$$
g_{0}^{e}-\frac{\left(g_{1}^{e}+\mu\right)^{2}}{4 g_{2}^{e}}=+g_{0}^{c} \text {. }
$$

One useful way to use and interpret this equation is to consider it as defining a boundary in the $\mu-\kappa^{2}$ plane that corresponds to the properties of the counterions. Within this diagram, a particular form of the relation between concentration and screening for a counterion defines a path in the diagram parametrized by the salt concentration $m$. Examples of this are presented in Fig.3. for certain polymer properties values, and different types of $\mu-\kappa^{2}$ relations.

At this point is very easy to explain the observed near independence of the transition on the molecular weight of the chains. Only the coefficients $g_{2}$ carry information on the molecular weight, but as we have seen $g_{2}^{c}$ drops out of the final equations, and in $g_{2}^{e}$ the molecular weight provides only an exponentially small correction to the dominant term given by a function of the screening length.

As shown in Fig.3, and as it can be seen from the fact that the equations defining the transition are quadratic in $\mu$, the expanded state splits into two regions, each with non-zero effective charge, but with opposite signs on each. This diagram is obtained using the approximations presented above, and a more precise determination of the lines of transition will involve consideration of the monovalent counterions, the finite amount of monomers, and the rod-like state obtained at very low screening. When these considerations are taken into account, the trajectory described by the added salt in the $\mu-\kappa^{2}$ plane will start within the expanded region, cross into the collapsed region (giving rise to the L1-L2 line), and continue there until crossing again, redissolving, into one of the branches with expanded states. The starting point of the trajectory, with zero multivalent salt added $m=0$, is located in the branch with natural charge, at a finite $\kappa$ value (due to the monovalent counterions), and at $\mu=\infty$. Figure 3 illustrates only the redissolution.

In Fig.3 we show three different curves defining the properties of the counterions: a pure Debye-Huckel case 
where the size of the counterions are neglected, a modified Debye-Huckel curve with $a=0.2$ (that is $a=0.2 b$ ), and a third curve that neglects the interactions between the counterions when they are free considers only the entropic term. The reversal of direction in the $\mu$ axis of the parametric curve for the Debye-Huckel cases occurs because of the onset of important favorable interactions between the free charges. Thus it is clear that the trajectory can end in the naturally charged branch of the expanded region since there are more free counterions there, and at the same time the repulsive intra-chain interactions are small. The overcharged branch is only accessible if the chemical potential is further reduced at the same time that the screening increases. For most cases with sensible sizes for the counterions the reversal in direction of the parametrized curve comes always before the redissolution, and thus the redissolution is into the naturally charged state.

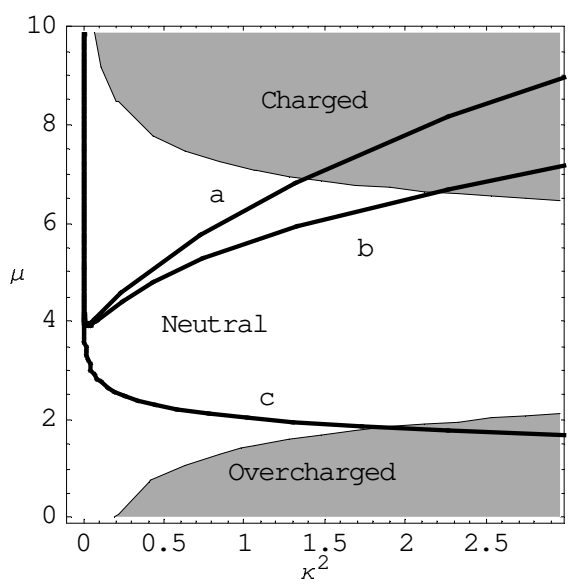

FIG. 3. Diagram of $\mu-\kappa^{2}$ showing the regions of expanded (gray) and collapsed (white) conformations. The solid curves give the relation of $\mu(\kappa)$ using the Full Debye-Huckel model for different size of the ions $a$. The first line (a) is for $a=0$, the second line (b) is for $a=0.2$ and the third curve (c) is for pure entropy (i.e., neglecting the electrostatic interactions of the multivalent ions in the solution, and mathematically equivalent to $a=\infty$ ). The three lines are indistinguishable at low concentrations (small values of $\kappa^{2}$ ).

It is interesting to note that at high enough salt concentrations and high valences, the salt might re-associate on its own. In this case the trajectory in the $\mu-\kappa^{2}$ plane has a different shape than that provided by the Debye-Huckel equation. Indeed, while the results from the Debye-Huckel model are know to provide good approximations for the free energy of the salt in solution they do not properly describe the individual dissociated species. The upturn of the chemical potential essentially indicates a phase transition for the salt/solvent system. In reality there is no phase transition but there is a finite a fraction of ion pairs formed in the solvent (even if they do not reassociate chemically). We have not presented here the curve in the $\mu-\kappa^{2}$ diagram when the association of multivalent ions in the solution is present, as is modeled, for example, in the classical Bjerrum model18, or more recently by Fisher, et al.26 This association gives large corrections to the Debye-Huckel law (for the free energy of the individual species of ions) and in our case provides a $\mu-\kappa$ curve that monotonically approaches a horizontal line (a limiting $\mu$ value), with increasing inverse screening length. These effects are particularly important for large valence salts as in our case and we will discuss them in more detail in a future publication 27 .

\section{COMPARISON WITH EXPERIMENTAL INFORMATION}

With the previously obtained solutions we now contrast our results with the currently known experimental situation. We consider the shape of the phase diagram, the precise location of the collapse transition and the predicted structure of the polymers in both of the phases.

It should be clear that we have already recovered the shape of the $B-C$ transition. Indeed, the (approximate) equation that define the transition does not have any dependence on the monomer concentration, and therefore produces a flat line in the $\phi-m$, or $\phi-\mu$ diagrams. The transition is defined by the choice of single chains to be in one of two states that must be in equilibrium with the surrounding environment. This environment, represented here by means of an effective chemical potential and screening length, depends strongly on the amount of multivalent salt and only very slightly on the polymer amount. The more precise expressions for the chemical potential recovers a small dependence in the polymer concentration.

There are two important ways in which important changes on the qualitative form of the diagram can arise. The addition of large amounts of monovalent salt introduces new features in the phase diagram, not show in the scheme of Fig. 1. In the introduction we only mentioned the effects on the $A-B$ transition, but further addition of monovalent salt, creates a different environment of the chains, and changes the transition points and the shape of the transition curve. Secondly, it is clear that as we continue increasing the amount of polymer in the solution, the interactions between the chains start to become important and the transition (if any) is a much more complicated phenomena. From the point of view of our theory it is clear that the increase in concentration of monovalent salt and monomers bring about a breakdown of the assumption that the chemical potential for the overcharging is dependent only on the amount of multivalent salt. The effective environment of the chain becomes more complicated and the chemical potential will now contain important terms coming form the concentrations of all species and from many-body interactions. 
Let us consider two concrete numerical examples. Experiments performed on polystyrene sulfonatet at room temperature with $N=4 * 10^{3}$, Bjerrum number of $B=2.87(b=0.5 \mathrm{~nm})$ and an effective ion radius $a / b=0.2$, show a transition line at a concentration of $m=0.2 M$ of $\mathrm{LaCl}_{3}$. With these parameters as input, our equation predicts redissolution at a salt concentration of $m=\beta .1 M$. Secondly, experiments on double stranded DNA2 with very large number of base pairs (we take $\left.N=10^{4}\right)$ and $B=4.2(b=.17 \mathrm{~nm})$ in spermine $(z=4), a=1$. obtained redissolution at $m=.1 M$, while our equations predict a transition at $m=0.04 M$. This compares favorably with the experiments, especially if we take into account the rough approximations done in the evaluation of the energies of the system.

According to the theory presented, in phase B, the polymer chains are almost neutral with a collapsed conformation. In phase $\mathrm{C}$, the chains are expanded and charged. This suggests, besides other techniques, to confirm these predictions by means of scattering experiments that test the structure of the chains, and of osmotic pressure measurements that can determine the amount of free counterions in eachstate (as it has been done for semidilute solutions 28 30).

\section{CONCLUSIONS}

The redissolution transition observed in multivalent induced precipitated polyelectrolytes with further addition of multivalent particles or salt was predicted extending a previously developed two state model to deal with large salt concentrations. The electrostatic energies of both, collapsed and expanded-coiled, states were computed considering the finite size of the ions and monomers condensed along the chains, and using a mean field approach for the non-condensed ions. We neglected the non-ionic short range interactions of the ions with the solvent, and assume zero monovalent salt.

We found that the redissolution is determined by the properties of the ionic solution. We calculated the effective charge of the chains for the different thermodynamic states of the chain.

\section{Acknowledgments}

We thank E. Raspaud and F. Livolant for useful discussions and for performing electrophoresis experiments 3 , M. Campos for computing electrostatic energies of dense finite size systems of charges, and P. A. Bernikowicz for computing the free energy of aggregates containing $p=1,2, \ldots$ chains. This work was sponsored by the National Science Foundation, grants DMR9807601 and DMR9632472.
${ }^{1}$ J. Widom, R. L. Baldwin: J. Mol. Biol. 144 (1980) 431 Biopolymers 22 (1983) 1595

${ }^{2}$ E. Raspaud, M. Olvera de la Cruz, J.-L. Sikorav, F. Livolant: Biophys. Jour. 74, 381 (1998)

${ }^{3}$ E. Raspaud, I. Chaperon, A. Leforestier, F. Livolant: Biophys. J. 77 (1999) 1547

${ }^{4}$ M. Olvera de la Cruz, L. Belloni, M. Delsanti, J. P. Dalbiez, O. Spalla, M. Drifford: J. Chem. Phys. 103, (1995) 5871

${ }^{5}$ J. Wittner, A. Johner J. F. Joanny: J. Phy. (paris) II 5 (1995) 635

${ }^{6}$ V. A. Bloomfield: Curr. Opin. Struct. Biol. 6 (1996) 334

${ }^{7}$ F. J. Solis, M. Olvera de la Cruz: J. Chem. Phys. 112 (2000) 2030

${ }^{8}$ T. T. Nguyen, I. Rouzina, B. I. Shklovskii: J. Chem. Phys. 112 (2000) 2562

${ }^{9}$ F. Livolant: Euro. J. Cell Biol. 33 (1984) 300.

${ }^{10}$ D. Jary, J. Lal, J.-L. Sikorav: Molecular Biology 321 (1998) 1

11 J. L. Sikorav, G. M. Church: J. Mol. Bio. 222 (1991) 1085 I. Chaperon, J.-L. Sikorav: Biopolymers 46 (1998) 195

12 S. Y. Park, D. Harries, W. M. Gelbart: Biophysical J. 75 (1998) 714

${ }^{13}$ P. Gonzales-Mozuelos, M. Olvera de la Cruz: J. Chem. Phys. 103 (1995) 3145

${ }^{14}$ G.S. Manning: J. Phys. Chem. 88 (1984) 6654

15 J. L. Barrat, J. F. Joanny: Adv. Chem. Phys. 94 (1996) 16 (1996).

${ }^{16}$ N. Lee and D. Thirumalai: pre-print cond-mat/9907199

${ }^{17}$ E. P. Wigner: Trans. Faraday Soc. 34 (1938) 678

${ }^{18}$ N. Bjerrum: Koninklinge Dans. Vidensk. Selsk. 7 (1926) 9. See: B. E. Conway: "Ionic Interactions and Activity Behavior of Electrolyte Solutions" section 7.2.3. in B. E. Conway, J. O’M. Bockris, E. Yeager: Comprehensive Treatise of Electrochemistry; v.5. New York: Plenum Press (1983).

${ }^{19}$ E. M. Mateescu, C. Jeppesen, P. Pincus: Europhys. Lett. 46 (1999) 493

${ }^{20}$ S. Y. Park, R. F. Bruinsma, W. M. Gelbart: Europhys. Lett. 46 (1999) 454

${ }^{21}$ I. Koltover, T. Salditt, C. R. Safinya: Biophys. J. 77 (1999) 915

22 P.S. Kuhn, Y. Levin, M.C. Barbosa: Physica A 274 (1999) 8

${ }^{23}$ E. Gurovitch, P. Sens: Phys. Rev. Lett. 82 (1999) 339

${ }^{24}$ M. H. Panckhurst, Aust. J. Chem. 15 (1962) 383 D. R. Rosseinsky: J. Chem. Soc. (1962) 785

${ }^{25}$ An elementary introduction to the Madelung constant appears in R. P. Feynman, R. B. Leighton, M. Sands: The Feynman lectures in Physics, Section II.8.3, Reading: Addison-Wesley 1964

${ }^{26}$ M.E. Fisher and Y. Levin: Phys. Rev. Lett. 71 (1993) 3826

${ }^{27}$ F. J. Solis, M. Olvera de la Cruz: unpublished

${ }^{28}$ I. Sabbagh, M. Delsanti, P. Lisieur: Eur. Phys. J. B 12 (1999) 253

29 B.D. Ermi, E. J. Amis: Macromolecules 31 (1998) 7378

${ }^{30}$ W. Essafi, F. Lafuma, C.E. Williams: Eur. Phys. J. B 9 (1999) 261 